Research Paper

\title{
Expression of the receptor activator of nuclear factor-kB ligand in peripheral blood mononuclear cells in patients with acute Charcot neuroarthropathy
}

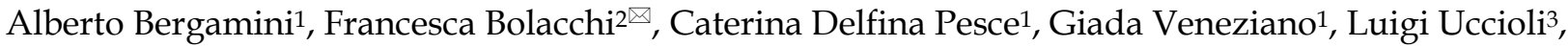 \\ Valentina Girardi², Laura De Corato2 ${ }^{2}$, Maria Teresa Mondillo², Ettore Squillaci² \\ 1. Department of Internal Medicine, Hematology/Oncology Unit, Tor Vergata University, Rome, Italy Department of Public Health and Cellular Biology, \\ University of Rome "Tor Vergata", Rome, Italy \\ 2. Department of Diagnostic and Molecular Imaging, Radiation Therapy and Interventional Radiology, University Hospital Tor Vergata, Rome, Italy \\ 3. Department of Systems Medicine, University of Rome Tor Vergata, Rome, Italy \\ $\triangle$ Corresponding author: Francesca Bolacchi, M.D., PhD., Department of Diagnostic and Molecular Imaging, Radiation Therapy and Interventional Radiology, \\ University Hospital Tor Vergata, Viale Oxford 8100133 Rome Italy. Tel. 06-20902374 Email: francesca.bolacchi@libero.it
}

(ㅇ Ivyspring International Publisher. Reproduction is permitted for personal, noncommercial use, provided that the article is in whole, unmodified, and properly cited. See http://ivyspring.com/terms for terms and conditions.

Received: 2015.12.02; Accepted: 2016.07.08; Published: 2016.10.20

\begin{abstract}
Introduction. The receptor activator of nuclear factor-kB (RANK), ligand (RANK-L) and osteoprotegerin (OPG) are implicated in the pathogenesis of acute Charcot neuroarthropathy $(\mathrm{CN})$.

Materials and Methods. This study aimed to investigate the expression of RANK-L and OPG in peripheral blood mononuclear cells (PBMC) from patients with acute $\mathrm{CN}$.

Results. We found that the expression of RANK-L was lower in patients with acute $C N$ as compared with diabetic control subjects and healthy control participants; whereas OPG expression was not detected in patients and in both control groups. RANK-L expression at the onset of disease was inversely correlated with the index of polyunsaturation (PUI), a bone marrow MRS-derived measurable index that allows evaluation of disease activity in acute $\mathrm{CN}$, and recovery time. Finally, the expression of RANK-L increased at the time of healing compared with the values found during the acute phase.

Conclusions. In conclusion, our preliminary data provide a first step in applying analysis of RANK-L expression in peripheral blood cells to the diagnosis of acute CN. Based on our data we also suggest that analysis of RANK-L expression could be a complementary tool that can be employed to obtain quantitative parameters that may help clinicians to monitor disease activity in patients with acute $\mathrm{CN}$.
\end{abstract}

Key words: Charcot neuroarthropathy, receptor activator of nuclear factor-kB, peripheral blood cells.

\section{Introduction}

Charcot neuroarthropathy $(\mathrm{CN})$ typically occurs in the foot/ankle of diabetic patients with sensory neuropathy and it is a common cause of morbidity in this population. This disease can lead to a severe amputation of feet: in such cases, osteomyelitis has been described as trigger mechanisms of developing $\mathrm{CN}$, thus an effective antibiotic therapy could have a positive role in the course of the disease. $\mathrm{CN}$ is early characterised by acute inflammation that may cause osteopenia, bone resorption and bone weakening which subsequently may lead to chronic bone alterations such as fractures, dislocations, instability and gross deformities ${ }^{1-3}$. Indeed, local inflammation is associated with the release of proinflammatory cytokines such as interleukin (IL)-1alpha and tumor necrosis factor (TNF)-alpha, which are known mediators of bone resorption via excess osteoclastic activity 4,5. These cytokines lead to an increased 
expression of the receptor activator of nuclear factor-kB (RANK) ligand (RANK-L). Its receptor (RANK) is expressed in the membrane of preosteoclasts. RANK-L stimulates the expression of nuclear factor (NF)-kB that, in turn, induces the maturation of precursor cells into mature osteoclasts. At the same time, NF-kB induces the glycoprotein osteoprotegerin (OPG), which acts as a decoy receptor for RANK-L to avoid excess osteolysis. The role of this pathway in acute $\mathrm{CN}$ pathogenesis is supported by the fact that the same RANK/RANK-L/OPG system is also involved in the process of medial arterial calcification a feature that is strongly associated with $\mathrm{CN}$ 6,7. Activated T- and B-cells are well-recognized sources of RANK-L and OPG ${ }^{8,9}$, and may contribute to pathological bone resorption seen in chronic inflammatory diseases such as rheumatoid arthritis (RA), periodontitis and inflammatory bowel disease 10-13. Acute $\mathrm{CN}$ is not associated with systemic inflammation ${ }^{14}$, however, peripheral monocytes from patients with acute $\mathrm{CN}$ may show pro-inflammatory changes ${ }^{15}$.

On the basis of these evidences, in this study the expression of RANK-L and OPG was investigated in peripheral blood mononuclear cells (PBMC) from patients with acute $\mathrm{CN}$ and correlated with clinical and radiological markers of disease activity. We found that the expression of RANK-L was lower in patients with acute $\mathrm{CN}$ as compared with diabetic control subjects and healthy control participants; whereas OPG expression was not detected in patients and in both control groups. RANK-L expression at the onset of disease was inversely correlated with the index of polyunsaturation (PUI), a bone marrow MRS-derived measurable index that allows evaluation of disease activity in acute $\mathrm{CN} \mathrm{16}$, and recovery time. Finally, the expression of RANK-L increased at the time of healing compared with the values found during the acute phase.

\section{Materials and Methods}

\section{Patients recruitment}

Nine diabetic patients with acute $\mathrm{CN}$ were prospectively enrolled in the study. Acute $\mathrm{CN}$ was defined based on the following clinical and MRI signs: unexplained, relatively painless, increasing swelling of a foot and ankle, skin temperature increase of at least $2^{\circ} \mathrm{C}$ compared with the contralateral foot (delta-T), clinical instability due to ligamentous injury/occult trauma, presence of typical bone marrow oedema on MRI. All patients were in stage 0, according to Eichenholtz stage system and were free of any structural bone or articular alteration, as documented by computed tomographic evaluation, active foot ulceration and/or signs of soft tissue infection. All patients had unilateral foot involvement as documented by MRI (no evidence of bone marrow oedema), as well as clinical evaluation. All patients displayed severe peripheral sensory polyneuropathy. The presence of peripheral neuropathy was assessed by the vibration perception threshold (VPT expressed in Volts) and diabetic neuropathy index (DNI). Peripheral neuropathy was defined by a VPT $\geq 25$ Volts and/or a positive DNI score $>2$ points ${ }^{17}$. Following the diagnosis of acute $\mathrm{CN}$, all patients underwent foot offloading and immobilisation by serial total contact casting with progression to removable cast walkers. Recovery was defined by the disappearance of bone marrow oedema as evidenced demonstrated on MRI using T2-weighted short tau inversion recovery (STIR) images. A group $(n=9)$ of diabetic patients with peripheral sensory polyneuropathy, without clinical and radiological evidence of the history of $\mathrm{CN}$ was also studied. Healthy controls $(n=9)$ with no evidence of diabetes mellitus (according to self-reported absence of antidiabetic medication), were also included. This study was conducted according to the principles expressed in the Declaration of Helsinki and approved by our institutional review board. Informed consent was obtained from all subjects before the performance of the study.

\section{RT-PCR}

Total RNA was isolated in patients and controls within 24h of meeting enrolment criteria using using RNeasy Plus Mini Kit (Qiagen, Hombrechtikon, Switzerland), inclusive of DNase-I digestion, and then ethanol precipitated. Quantitation of isolated RNA was performed by spectrophotometric determination (Gene Quant II, Pharmacia, Uppsala, Sweden). Total RNA was reverse-transcribed to cDNA using Omniscript RT Kit (Qiagen): RNA (1microgram) was added to one reaction tube containing 2microliters 10x Buffer RT, 2microliters dNTP Mix (5mM each dNTP), 2microliters Oligo dT primer (10microM), 2microliters Random hexamers (100microM), 1microliter Qiagen-RNase inhibitor (10 units/microl), 1microliter Omniscript Reverse Transcriptase (4 units/microl), variable amount of RNase-free water in a total reaction volume of 20 microliter. Incubation conditions were $60 \mathrm{~min}$ at $37^{\circ} \mathrm{C}$. Serial dilutions of cDNA were amplified by PCR using AmpliTaq Gold 360 DNA Polymerase (Applied Biosystems): cDNA was added to one reaction tube containing 5microliters 10x AmplTaq Gold 360 Buffer, 5 microliters $25 \mathrm{mM}$ Magnesium Chloride, 4 microliters dNTP Mix (2.5mM each dNTP), 1microliter of each primer (25microM), 0.25microliters AmpliTaq Gold 
360 DNA Polymerase (5 units/microliter), PCR-grade water in a total reaction volume of 50 microliters. The reaction was performed on GeneAmp PCR System 9700 (Applied Biosystems). Human primers specific for mRNA from RANK-L and OPG genes were used in PCR, beta-actin was the housekeeping gene selected as internal standard. The primers used were: RANK-L forward, 5'-CAGATGGATCCTAATAGA AT-3', RANK-L reverse, 5'-ATGGGAACCAGA TGGGATGTC-3', OPG forward, 5'-ATGAACAA GTTGCTGTGCTG-3', OPG reverse, 5'-GCAGAACT CTATCTCAAGGTA-3', beta-actin forward, 5' -CGTA CCACTGGCATCGTGAT-3', beta-actin reverse, 5'-GTGTTGGCGTACAGGTCTTTG-3'. Thermal profile to amplify DNA was: initial denaturation at $95^{\circ} \mathrm{C}$ for $10 \mathrm{~min}$ followed by a selected number of cycles consisting of denaturation at $95^{\circ} \mathrm{C}$ for $1 \mathrm{~min}$, annealing (at temperature depending on $\mathrm{T}$ melting temperature of each primer pair) for $30 \mathrm{sec}$, extension at $72^{\circ} \mathrm{C}$ for $1 \mathrm{~min}$, with a final extension at $72^{\circ} \mathrm{C}$ for 7 $\mathrm{min}$. The annealing temperature was: $54^{\circ} \mathrm{C}$ for OPG, $46^{\circ} \mathrm{C}$ for RANKL, $58^{\circ} \mathrm{C}$ for beta-actin. The number of cycles was: 35 for RANK-L, 50 for OPG, 21 for beta-actin. The length of the amplification product was: 324 bp for RANK-L, 354 bp for OPG, 452 bp for beta-actin. The absence of DNA contamination in the RNA preparation was verified performing HLA-DQa 1 locus PCR amplification (forward primer GH26, 5-GTGCTGCAGGTGTAAACTTGTACCAG-3', reverse primer GH27, 5'-CACGGATCCGGTA GCAGCGGTAGAGTTG-3', annealing temperature $60^{\circ} \mathrm{C}$, size of product $242 \mathrm{bp}$, or $239 \mathrm{bp}$ from some alleles). $10 \mathrm{ml}$ of the amplification product from each PCR were separated on $1.8 \%$ agarose gel, stained with ethidium bromide and visualized by UV irradiation. Ethidium bromide bands were acquired by scanning and quantified by image analysis with Multi-Analyst/PC (PC software for Bio-Rad's Image Analysis Systems Version 1.1.)

\section{MR Examinations}

MRS and MRI were performed using a 3-T scanner system (Achieva, Philips Medical Systems, Best, the Netherlands). Presence or absence of bone marrow oedema within different bones of the foot and ankle was determined using T2-weighted short tau inversion recovery (STIR) images acquired in the coronal, axial and sagittal planes. Spectroscopic data were obtained as previously described using a single-voxel point-resolved spectroscopic sequence (PRESS) as previously described ${ }^{16}$. In brief, the PRESS sequence was water suppressed using a selective excitation pulse to crush the water signal. The voxel (volume of interest, VOI) size was $1.3 \times 1.3 \times 1.3 \mathrm{~cm} 3$. Fully automated frequency determination, power optimization, and shimming phases were performed in the VOI. To confirm the reproducibility of the measurements, three sets of spectra acquisition were repeated three times each in four 4 patients and six 6 control subjects. Data were analyzed with using the jMRUI v4.0 software package ${ }^{18}$. Metabolites to be estimated were defined with a reference database of known peaks ${ }^{19}$. The acquired spectra were analyzed with using the AMARES algorithm. The metabolite ratio index of poly-unsaturation (PUI) was defined as previously described ${ }^{16}$.

\section{Results}

\section{Clinical outcomes}

The general clinical characteristics of the different patient groups are presented in Table 1. The mean age, male to female ratio were similar between acute $\mathrm{CN}$ patients, and diabetic control subjects ( $p>0.05$ and $p>0.05$, respectively) or healthy control participants ( $p>0.05$ and $p>0.05$, respectively). Also, the time since onset of diabetes and percentage of Hba1c were not different between acute $\mathrm{CN}$ patients, and diabetic control subjects $(p>0.05$ and $p>0.05$, respectively). All diabetic patients were on insulin therapy. The lesion site in acute $\mathrm{CN}$ patients was hind-foot, $\mathrm{n}=6$; mid-foot, $\mathrm{n}=3$ and the mean healing time on MRI was $8.14 \pm 3.7$ months.

Table 1: General characteristics of study patients

\begin{tabular}{llll}
\hline & Charcot & Diabetes & $\begin{array}{l}\text { Healthy } \\
\text { controls }\end{array}$ \\
\hline Age (years, mean \pm SD) & $54.4 \pm 4.5$ & $60.11 \pm 4.3$ & $48.89 \pm 5.1$ \\
Sex (male:female) & $3: 6$ & $4: 5$ & $5: 4$ \\
Type1:type2 diabetes & $6: 3$ & $5: 4$ & n.a. \\
$\begin{array}{l}\text { Time since onset of diabetes } \\
\text { (years, mean } \pm \text { SD) }\end{array}$ & $18.34 \pm 11.3$ & $22.7 \pm 12.5$ & n.a. \\
Hba1c (\%, mean \pm SD) & $7.4 \pm 3.1$ & $8.1 \pm 2.2$ & n.a. \\
\hline Note. n.a.= not applicable & & &
\end{tabular}

\section{RANK-L and OPG expression in PBMC}

Semi-quantitative RT-PCR was performed on total RNA extracted from PBMC in acute $\mathrm{CN}$ patients, diabetic control subjects and healthy control participants. As shown in Figure 1, RANK-L expression was detected in the totality of patients and controls but was significantly lower in patients as compared to controls, as demonstrated by densitometric evaluation of the amplification products from PCR. No significant differences in RANK-L expression were documented between diabetic control subjects and healthy control participants. Abundant OPG expression was evidenced in the control positive cell line U937 but, at 
variance with RANK-L, no OPG expression was detected in PBMC from patients and controls.

\section{RANK-L expression correlates with PUI and recovery time}

We then investigated whether RANK-L expression in acute $\mathrm{CN}$ patients was correlated with PUI and healing time on MRI. Pearson's correlation analysis showed RANK-L expression was inversely correlated with PUI levels ( $\mathrm{r}=-0.7072,95 \%$ confidence interval $=-0.9331$ to $-0.0810, P=0.033$ ) and recovery time $(\mathrm{r}=-0.7878,95 \%$ confidence interval $=-0.9532$ to $-0.2592, P=0.011)$ (Fig. 2).

\section{Recovery from acute $\mathbf{C N}$ is associated with increase of RANK-L expression}

RANK-L expression was analyzed in six patients during the acute phase of $\mathrm{CN}$ and at time of bone marrow oedema disappearance (Fig. 3). Densitometric evaluation of the amplification

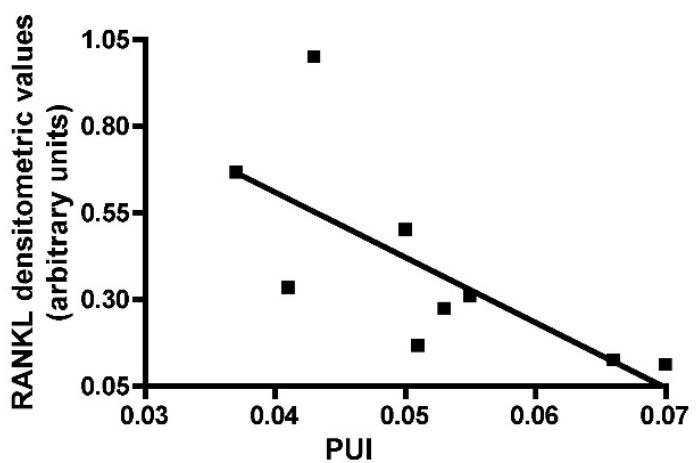

products from PCR did not show a significant difference of RANK-L expression between the two time points; however, in all patients the expression of RANK-L increased at the time of healing compared with the values found during the acute phase.

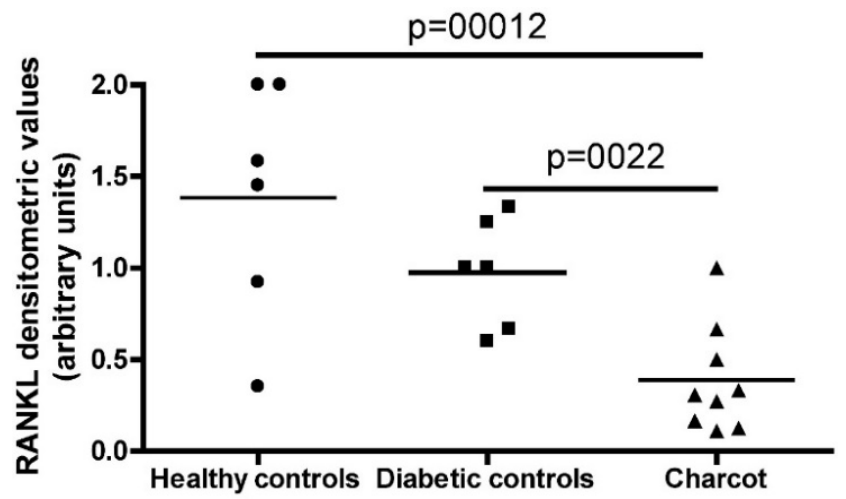

Figure 1. RT-PCR RANK-L expression in the 3 typologies of subjects investigates: Healthy controls, Diabetic controls and Charcot patients.

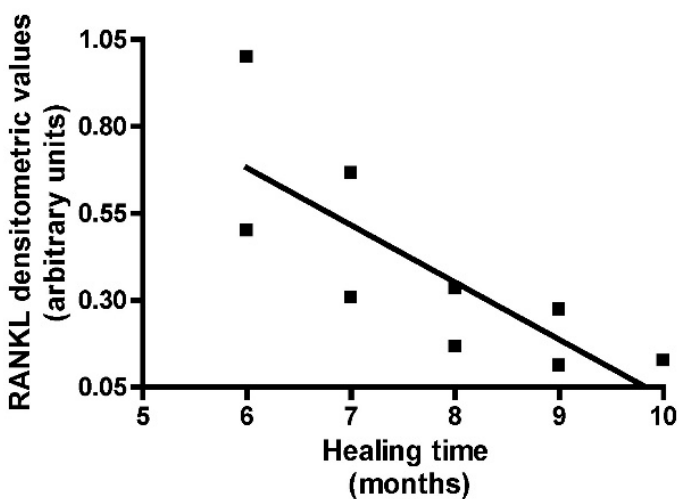

Figure 2. RT-PCR RANK-L expression in acute Charcot patients evaluated on the basis of the index of polyunsaturation (PUI) and of the healing time on MRI.



Figure 3. RANK-L expression analyzed in Charcot patients in 2 time-points: during the acute phase of Charcot neuropathy and at time of bone marrow oedema disappearance.

\section{Discussion}

The involvement of RANK-L-mediated osteoclastic resorption in acute $\mathrm{CN}$ is supported by several studies 6,7 . The adaptive immune system potently impacts the skeleton via a convergence of immune cells and cytokine effectors, mediating critical functions in both organ systems and forming the "immuno-skeletal interface". Under inflammatory conditions both $\mathrm{B}$ and $\mathrm{T}$ cells can be considerable sources of RANK-L and may contribute to pathological bone resorption ${ }^{10-13}$. However, in acute $\mathrm{CN}$ the local inflammatory response related to increased pro-inflammatory cytokine secretion is not associated with a systemic inflammatory syndrome (14). Here we found that the expression of RANK-L was lower in PBMC from patients with acute $\mathrm{CN}$ as compared with diabetic control subjects and healthy controls. These data suggest the existence of a compensatory immunoregulatory mechanisms to potentially limit bone resorption during acute $\mathrm{CN}$. Down-modulation of RANK-L expression could be obtained by either reduced production of cytokines such as IL-18, IL-1beta and TNF-alpha, that increase 
RANK-L production by lymphocytes 20 , or by increased secretion of cytokines, such as IL-27, able to inhibit expression of RANK-L on $\mathrm{T}$ cells ${ }^{21-24}$. In contrast to RANK-L, no OPG expression was detected in cells from patients and controls. This is not surprising since lymphocytes express OPG only upon activation in response to antigen challenge, which is not a pathogenic mechanism of acute CN. Notably, absence of OPG expression by PBMC is consistent with the intense bone resorption that characterizes the acute phase of $\mathrm{CN}$.

Acute $\mathrm{CN}$ typically presents with acute or sub-acute inflammation of the foot. Pain may or may not be present, depending on the presence of nerve damage ${ }^{5}$. There is neither fever nor any major biological inflammatory syndrome, and this clinical presentation has often led to inaccurate or delayed diagnosis, resulting in progression to the chronic phase with irreversible deformation. Early recognition of $\mathrm{CN}$ can save a long period of suffering for the patient, high hospital costs and ultimately amputation. However, at this stage of disease standard radiography often can not distinguish acute $\mathrm{CN}$ from other conditions. Radioisotope technetium (TC-99m) bone scintigraphy has good sensitivity but poor specificity for osseous pathology 25 . MRI is able to evidence inflammation in the bone and in the adjacent soft tissues, but it is quite expensive and requires a high degree of expertise by the examiner ${ }^{26}$. The data we present here suggest that evaluation of RANK-L expression in peripheral blood cells may represent a possible new diagnostic tool. However, differential diagnosis of acute $\mathrm{CN}$ is to be made with infections (osteomyelitis, cellulitis, septic arthritis) and inflammations (i.e. gout). We did not evaluate RANK-L expression in subjects with infection or inflammation other than $\mathrm{CN}$, thus, future work should focus, other than on confirmatory analysis, on discrimination of infection/inflammation vs. CN.

$\mathrm{Up}$ to now the evaluation of acute $\mathrm{CN}$ patients has relied on local inflammatory signs such as skin temperature, swelling and erythema 5,27,28. Local clinical signs are useful to guide the physician to shift from the cast to other pressure relieving devices. However, they are affected by poor specificity and reproducibility and their utility in providing a measure of disease activity level has been recently questioned 29,30. The lack of specific criteria and objective indices able to assess the disease activity during follow-up, in addition to being a hindrance to the implementation of current physical therapeutic options has also limited research concerning pharmacological treatments for acute CN. Recently, MRI has been introduced in the evaluation of acute $\mathrm{CN}$. In particular, the disappearance of bone marrow oedema as evaluated on STIR images has proved useful in assessing disease recovery ${ }^{31}$. However, during the period in between disease onset (presence of bone marrow oedema) and recovery (absence of bone marrow oedema) the regression of bone marrow oedema is difficult to quantify ${ }^{32}$. The use of contrast enhanced MRI has been advocated 32,34. However, repeated contrast enhanced examinations are not always feasible in diabetic patients ${ }^{34}$. In this clinical contest, a quantitative non-invasive index would be highly desirable to monitor disease activity. In this regard, we show here that the degree of RANK-L expression in PBMC from acute $\mathrm{CN}$ patients correlates with the intensity of bone marrow inflammation, as assessed by MRS. Also, RANK-L expression was significantly lower in patients whose marrow edema took longer to resolve. Finally, after bone marrow oedema disappearance the expression of RANK-L evaluated on a single patient basis increased with respect to the values obtained at the onset of disease.

\section{Conclusions}

In conclusion, our preliminary data provide a first step in applying analysis of RANK-L expression in peripheral blood cells to the diagnosis of acute $\mathrm{CN}$. RANK-L expression could be related to a wide range of degenerative bone pathologies, such as rheumatoid arthritis, thus a proper differential diagnosis must be performed. The pathogenesis of $\mathrm{CN}$ remains uncertain and however, based on our data, we suggest that analysis of RANK-L expression could be a complementary tool that can be employed to obtain quantitative parameters that may help clinicians to monitor disease activity in patients with acute $\mathrm{CN}$.

\section{Authors' Contributions}

\section{Authors equally contributed to this study.}

\section{Competing Interests}

The authors have declared that no competing interest exists.

\section{References}

1. Nielson DL, Armstrong DG. The natural history of Charcot's neuroarthropathy. Clin Podiatr Med Surg. 2008; 25:53-62

2. Chantelau E. The perils of procrastination: effects of early vs. delayed detection and treatment of incipient Charcot fracture. Diabet Med. 2005; 22:1707-1712

3. Greenstein A, Jarrett SJ, McGonagle D. Acute neuropathic joint disease: a medical emergency? Diabetes Care. 2005; 28:2962-2964

4. Baumhauer JF, O'Keefe RJ, Schon LC, Pinzur MS. Cytokine-induced osteoclastic bone resorption in charcot arthropathy: an immunohistochemical study. Foot Ankle Int. 2006; 27:797-800

5. Petrova NL, Moniz C, Elias DA, Buxton-Thomas M, Bates M, Edmonds ME. Is there a systemic inflammatory response in the acute charcot foot? Diabetes Care. 2007; 30:997-998

6. Sinha S, Munichoodappa CS, Kozak GP. Neuro-arthropathy (Charcot joints) in diabetes mellitus (clinical study of 101 cases). Medicine (Baltimore). 1972; 51:191-210. 
7. Clouse ME, Gramm HF, Legg M, Flood T. Diabetic osteoarthropathy. Clinical and roentgenographic observations in 90 cases. Am J Roentgenol Radium Ther Nucl Med. 1974;121:22-34

8. Wong BR, Josien R, Lee SY, et al. TRANCE (Tumor necrosis factor [TNF]-related Activation-induced Cytokine), a new TNF family member predominantly expressed in t cells, is a dendritic cell-specific survival factor. The Journal of Experimental Medicine. 1997; 186:2075-2080

9. Yun TJ, Chaudhary PM, Shu GL, et al. OPG/FDCR-1, a TNF receptor family member, is expressed in lymphoid cells and is up-regulated by ligating CD40. J Immunol. 1998; 161:6113-6121

10. Haynes DR, Crotti TN, Loric M, Bain GI, Atkins GJ, Findlay DM. Osteoprotegerin and receptor activator of nuclear factor kappaB ligand (RANKL) regulate osteoclast formation by cells in the human rheumatoid arthritic joint. Rheumatology (Oxford). 2001; 40:623-630.

11. Theill LE, Boyle WJ, Penninger JM. RANK-L and RANK: T cells, bone loss, and mammalian evolution. Annu Rev Immunol. 2002; 20:795-823

12. Teng YT, Nguyen H, Gao X, Kong YY, Gorczynski RM, Singh B, Ellen RP, Penninger JM. Functional human T-cell immunity and osteoprotegerin ligand control alveolar bone destruction in periodontal infection. J Clin Invest. 2000; 106:R59-67

13. Jeffcoate WJ. Vascular calcification and osteolysis in diabetic neuropathy, is RANK-L the missing link? Diabetologia. 2004; 47:1488-1492

14. Byrne FR, Morony S, Warmington K, et al. CD4 + CD45RBHi T cell transfer induced colitis in mice is accompanied by osteopenia which is treatable with recombinant human osteoprotegerin. Gut. 2005; 54:78-86

15. Uccioli L, Sinistro A, Almerighi C, Ciaprini C, Cavazza A, Giurato L, Ruotolo V, Spasaro F, Vainieri E, Rocchi G, Bergamini A. Proinflammatory modulation of the surface and cytokine phenotype of monocytes in patients with acute Charcot foot. Diabetes Care. 2010; 33:350-355

16. Bolacchi F, Uccioli L, Masala S, Giurato L, Ruotolo V, Meloni M, Baffari E, Cinelli E, Cadioli M, Squillaci E, Simonetti G, Bergamini A. Proton magnetic resonance spectroscopy in the evaluation of patients with acute Charcot neuro-osteoarthropathy. Eur Radiol. 2013; 23:2807-2813

17. Fedele D, Comi G, Coscelli C, Cucinotta D, Feldman EL, Ghirlanda G et al. A multicenter study on the prevalence of diabetic neuropathy in Italy. Italian Diabetic Neuropathy Committee. Diabetes Care. 1997; 20:836-843

18. Naressi A, Couturier C, Castang I, de Beer R, Graveron-Demilly D. Java-based graphical user interface for MRUI, a software package for quantitation of in vivo/medical magnetic resonance spectroscopy signals. Comput Biol Med. $2001 ; 31: 269-286$

19. Vanhamme L, van den Boogaart A, Van Huffel S. Improved method for accurate and efficient quantification of MRS data with use of prior knowledge. J Magn Reson. 1997; 129:35-43.

20. Dai SM, Nishioka K, Yudoh K. Interleukin (IL) 18 stimulates osteoclast formation through synovial $\mathrm{T}$ cells in rheumatoid arthritis: comparison with IL1 beta and tumour necrosis factor alpha. Ann Rheum Dis. 2004; 63:1379-1386

21. Inchingolo F, Tatullo M, Marrelli M, Inchingolo AM, Inchingolo AD, Dipalma G, Flace P, Girolamo F, Tarullo A, Laino L, Sabatini R, Abbinante A, Cagiano $R$. Regenerative surgery performed with platelet-rich plasma used in sinus lift elevation before dental implant surgery: an useful aid in healing and regeneration of bone tissue. Eur Rev Med Pharmacol Sci. 2012; 16(9):1222-1226

22. Tatullo M, Marrelli M, Cassetta M, Pacifici A, Stefanelli LV, Scacco S, Dipalma G, Pacifici L, Inchingolo F. Platelet Rich Fibrin (P.R.F.) in reconstructive surgery of atrophied maxillary bones: clinical and histological evaluations. Int J Med Sci. 2012; 9:872-880

23. Marrelli M, Tatullo M. Influence of PRF in the healing of bone and gingival tissues. Clinical and histological evaluations. Eur Rev Med Pharmacol Sci. 2013; 17:1958-1962.

24. Kamiya S1, Okumura M, Chiba $Y$, Fukawa T, Nakamura C, Nimura N, Mizuguchi J, Wada S, Yoshimoto T. IL-27 suppresses RANKL expression in CD4+ T cells in part through STAT3. Immunol Lett. 2011; 138:47-53

25. Inchingolo F, Tatullo M, Marrelli M, Inchingolo AM, Picciariello V, Inchingolo AD, Dipalma G, Vermesan D, Cagiano R. Clinical trial with bromelain in third molar exodontia. Eur Rev Med Pharmacol Sci. 2010; 14:771-774

26. Ledermann HP, Morrison WB. Differential diagnosis of pedal osteomyelitis and diabetic neuroarthropathy: MR Imaging. Semin Musculoskelet Radiol. 2005; 9:272-283

27. Morrison WB, Ledermann HP. Work-up of the diabetic foot. Radiol Clin North Am. 2002; 40:1171-1192

28. Tomas MB, Patel M, Marwin SE, Palestro CJ. The diabetic foot. Br J Radiol. 2000; 73:443-450

29. Boyko EJ, Ahroni JH, Stensel VL. Skin temperature in the neuropathic diabetic foot. J Diabetes Complications. 2001; 15:260-264

30. Armstrong DG, Lavery LA. Monitoring neuropathic ulcer healing with infrared dermal thermometry. J Foot Ankle Surg. 1996; 35:335-338

31. Schlossbauer T, Mioc T, Sommerey S, Kessler SB, Reiser MF, Pfeifer KJ. Magnetic resonance imaging in early stage charcot arthropathy: correlation of imaging findings and clinical symptoms. Eur J Med Res. 2008; 13:409-414

32. Zampa V, Bargellini I, Rizzo L, Turini F, Ortori S, Piaggesi A, Bartolozzi C. Role of dynamic MRI in the follow-up of acute Charcot foot in patients with diabetes mellitus. Skeletal Radiol. 2011; 40:991-999

33. Inchingolo $\mathrm{F}$, Tatullo $\mathrm{M}$, Abenavoli FM, Marrelli $\mathrm{M}$, Inchingolo $\mathrm{AD}$ Inchingolo AM, Dipalma G. Non-Hodgkin lymphoma affecting the tongue: unusual intra-oral location. Head Neck Oncol. 2011; 3:1
34. Andersen PE. Patient selection and preparation strategies for the use of contrast material in patients with chronic kidney disease. World J Radiol. 2012; 4:253-257 